\title{
Boundedness and compactness of a class of integral operators with power and logarithmic singularity when $p \leq q$
}

Akbota Abylayeva ${ }^{1 *}$ (D) Ryskul Oinarov ${ }^{1}$ and Bolat Seilbekov ${ }^{1}$

"Correspondence:

abylayeva_b@mail.ru

'Faculty of Mechanics and

Mathematics, L.N. Gumilyov

Eurasian National University,

Satpaev str. 2, Nur-Sultan, 010008 ,

Kazakhstan

\section{Springer}

\begin{abstract}
In this paper, necessary and sufficient conditions for the boundedness and compactness of one class of integral operators with power and logarithmic singularities in weighted Lebesgue spaces are obtained.
\end{abstract}

Keywords: Boundedness; Compactness; Weight function; Logarithmic singularity

\section{Introduction}

Let $I=(0, \infty)$ and let $v, u$ be almost everywhere positive and locally integrable functions on the interval $I$.

Let $1<p, q<\infty$, and $p^{\prime}=\frac{p}{p-1}$. Let us denote by $L_{p, v} \equiv L_{p}(v, I)$ the set of measurable functions $f$ on $I$ for which

$$
\|f\|_{p, v}=\left(\int_{0}^{\infty}|f(x)|^{p} v(x) d x\right)^{\frac{1}{p}}<\infty .
$$

Let $W$ be a positive, strictly increasing, and locally absolutely continuous function on the interval $I$. Let $\frac{d W(x)}{d x}=w(x)$ for almost all $x \in I$.

Consider the operator

$$
T_{\alpha, \beta} f(x)=\int_{0}^{x} \frac{\left(\ln \frac{W(x)}{W(x)-W(s)}\right)^{\beta} u(s) f(s) w(s) d s}{(W(x)-W(s))^{1-\alpha}}, \quad x \in I,
$$

where $\alpha>0, \beta \geq 0$.

When $\beta=0$, the operator $T_{\alpha, \beta}$ has the form

$$
T_{\alpha} f(x)=\int_{0}^{x} \frac{u(s) f(s) w(s) d s}{(W(x)-W(s))^{1-\alpha}}
$$

which is called the fractional integration operator of the function $f$ over the function $W$ for $u \equiv 1$.

(c) The Author(s) 2022. This article is licensed under a Creative Commons Attribution 4.0 International License, which permits use, sharing, adaptation, distribution and reproduction in any medium or format, as long as you give appropriate credit to the original author(s) and the source, provide a link to the Creative Commons licence, and indicate if changes were made. The images or other third party material in this article are included in the article's Creative Commons licence, unless indicated otherwise in a credit line to the material. If material is not included in the article's Creative Commons licence and your intended use is not permitted by statutory regulation or exceeds the permitted use, you will need to obtain permission directly from the copyright holder. To view a copy of this licence, visit http://creativecommons.org/licenses/by/4.0/. 
Operator (1.2) becomes the Riemann-Liouville fractional integration operator for $u \equiv$ $1, W(x)=x$, which were investigated in papers [1-4]. We obtain the Hadamard fractional integration operator from (1.2) for $u \equiv 1, W(x)=\ln x$.

Further, we assume that $W$ is nonnegative on $I$ and $\lim _{x \rightarrow 0^{+}} W(x)=0$.

The boundedness and compactness of operator (1.2) from $L_{p, w}$ to $L_{q, v}$ is obtained in the paper [5] for $\alpha>\frac{1}{p}, 1<p \leq q<\infty$, and $0<q<p<\infty$. When $\alpha>1$, the results follow from the results in [6]. A criterion for the boundedness and compactness of the dual operator (1.2), when the parameters satisfy the same conditions, was obtained in the paper [7]. The boundedness and compactness of operator (1.2) were obtained in the paper [8] when the upper limit of the integral is a function. When $\beta=1$ and $W(x)=x$ in (1.1), two-sided estimates have been obtained in the paper [9].

The main goal of the paper is to establish the criteria for the boundedness and compactness of operator (1.1) from $L_{p, w}$ to $L_{q, v}$ for the following relations of the space parameters $1<p \leq q<\infty$.

The work is organized as follows. The next section contains the necessary materials to confirm the main results, which are presented in the third and fourth sections. In the third section, we have proved the boundedness of operator (1.1), and the compactness of the operator is proved in the fourth section. The last section contains the corollaries.

Agreements. The uncertainty of the form $0 \cdot \infty$ is considered to be zero. We will write $A \ll B$ or $B \ll A$ if there is a number $c>0$ and $A \leq c B$. The relation $A \approx B$ means $A \ll B$ and $A \gg B . Z$ is the set of integers, and $\chi_{(a, b)}$ is the characteristic function of the interval $(a, b) \subset I$.

\section{Auxiliary statements}

Consider the Hardy operator

$$
H f(x)=\varphi(x) \int_{0}^{x} u(s) f(s) w(s) d s
$$

from $L_{p, w}$ to $L_{q, v}$, where $\varphi$ is a nonnegative measurable function on $I$.

Theorem 5 of the book [10] implies the following theorem.

Theorem A Let $1<p \leq q<\infty$. Then the Hardy operator $H$ is bounded from $L_{p, w}$ to $L_{q, v}$ if and only if

$$
A=\sup _{z>0}\left(\int_{z}^{\infty} \varphi^{q}(x) v(x) d x\right)^{\frac{1}{q}}\left(\int_{0}^{z} u^{p^{\prime}}(s) w(s) d s\right)^{\frac{1}{p^{\prime}}}<\infty ;
$$

moreover $\|H\| \approx A$, where $\|H\|$ is the norm of the operator $H$ from $L_{p, w}$ to $L_{q, v}$.

Now let us consider the properties of the function $\ln \frac{W(x)}{W(x)-W(s)}$ :

$$
\frac{W(s)}{W(x)-W(s)}>\ln \frac{W(x)}{W(x)-W(s)}=\int_{0}^{x} \frac{w(s) d s}{W(x)-W(s)}>\frac{W(s)}{W(x)}, \quad x>s>0 .
$$

The function $\frac{1}{W(s)} \cdot \ln \frac{W(x)}{W(x)-W(s)}$ increases with respect to $s \in(0, x)$. Indeed

$$
\frac{\partial}{\partial s}\left(\frac{1}{W(s)} \cdot \ln \frac{W(x)}{W(x)-W(s)}\right)=\frac{w(s)}{W^{2}(s)}\left(\frac{W(s)}{W(x)-W(s)}-\ln \frac{W(x)}{W(x)-W(s)}\right)>0
$$

for $s \in(0, x)$. 


\section{Boundedness of the operator $T_{\alpha, \beta}$}

The main result of this section is the following.

Theorem 3.1 Let $0<\alpha<1, \frac{1}{\alpha}<p \leq q<\infty$, and $\beta \geq 0$. Let the function $u$ be nonincreasing on I. Then the operator $T_{\alpha, \beta}$, defined by formula (1.1), is bounded from $L_{p, w}$ to $L_{q, v}$ if and only if

$$
A_{\alpha, \beta}=\sup _{z>0}\left(\int_{z}^{\infty} v(x) W^{q(\alpha-\beta-1)}(x) d x\right)^{\frac{1}{q}}\left(\int_{0}^{z} W^{\beta p^{\prime}}(s) u^{p^{\prime}}(s) w(s) d s\right)^{\frac{1}{p^{\prime}}}<\infty ;
$$

moreover $\left\|T_{\alpha, \beta}\right\| \approx A_{\alpha, \beta}$, where $\left\|T_{\alpha, \beta}\right\|$ is the norm of operator (1.1) from $L_{p, w}$ to $L_{q, v}$.

Proof of Theorem 3.1 Necessity. Let operator (1.1) be bounded from $L_{p, w}$ to $L_{q, v}$. Using the properties of the function $\ln \frac{W(x)}{W(x)-W(s)}$ for $x>s>0$, we have

$$
\frac{1}{(W(x)-W(s))^{1-\alpha}} \geq \frac{1}{(W(x))^{1-\alpha}} \quad \text { for almost all } x \in I .
$$

Substituting the obtained relations in the expressions of operator (1.1) for $f \geq 0$, we obtain

$$
T_{\alpha, \beta} f(x) \geq W^{\alpha-\beta-1}(x) \int_{0}^{x} W^{\beta}(s) u(s) f(s) w(s) d s \equiv H_{\alpha, \beta} f(x) .
$$

The boundedness of the operator $T_{\alpha, \beta}$ from $L_{p, w}$ to $L_{q, v}$ implies the boundedness of the Hardy operator $H_{\alpha, \beta}$ from $L_{p, w}$ to $L_{q, v}$ and $\left\|T_{\alpha, \beta}\right\| \gg\left\|H_{\alpha, \beta}\right\|$. Then, by Theorem A, the value of $A_{\alpha, \beta}<\infty$ and for the norm $\left\|H_{\alpha, \beta}\right\|$ of the operator $H_{\alpha, \beta}$ there is an estimate $A_{\alpha, \beta} \ll$ $\left\|H_{\alpha, \beta}\right\|$. Then, by virtue of (3.1),

$$
A_{\alpha, \beta} \ll\left\|T_{\alpha, \beta}\right\| .
$$

Sufficiency. Let $A_{\alpha, \beta}<\infty$. Since $W$ is a strictly increasing continuous function such that $\lim _{x \rightarrow 0^{+}} W(x)=0$, then for $k \in Z$ define $x_{k}=\sup \left\{x: W(x) \leq 2^{k}, x \in I\right\}$.

Let $k_{\infty}=\inf \left\{k \in Z: \sup _{x>0} W(x) \leq 2^{k}\right\}$. Then $0<x_{k}<x_{k+1}$ for $k+1 \leq k_{\infty}$. Then, without limiting generality, we put $k_{\infty}=\infty$. Then $I=\bigcup_{k \in Z}\left[x_{k}, x_{k+1}\right)$. Let $f \geq 0$.

We have

$$
\begin{aligned}
\left\|T_{\alpha, \beta} f\right\|_{q, v}^{q} & =\sum_{k} \int_{x_{k}}^{x_{k+1}} v(x)\left(\int_{0}^{x} \frac{\left(\ln \frac{W(x)}{W(x)-W(s)}\right)^{\beta}}{(W(x)-W(s))^{1-\alpha}} u(s) f(s) w(s) d s\right)^{q} d x \\
& =\sum_{k} \int_{x_{k}}^{x_{k+1}} v(x)\left[\left(\int_{0}^{x_{k-1}}+\int_{x_{k-1}}^{x}\right) \frac{\left(\ln \frac{W(x)}{W(x)-W(s)}\right)^{\beta}}{(W(x)-W(s))^{1-\alpha}} u(s) f(s) w(s) d s\right]^{q} d x \\
& \ll J_{1}+J_{2} .
\end{aligned}
$$

Now we estimate $J_{1}$ and $J_{2}$ separately.

$$
J_{1}=\sum_{k} \int_{x_{k}}^{x_{k+1}} v(x)\left(\int_{0}^{x_{k-1}} \frac{\left(\ln \frac{W(x)}{W(x)-W(s)}\right)^{\beta}}{(W(x)-W(s))^{1-\alpha}} u(s) f(s) w(s) d s\right)^{q} d x
$$


(using the monotonicity of the function $\left.\frac{1}{W(s)} \ln \frac{W(x)}{W(x)-W(s)}\right)$

$$
\begin{aligned}
& =\sum_{k} \int_{x_{k}}^{x_{k+1}} v(x)\left(\int_{0}^{x_{k-1}} \frac{\left(\frac{1}{W(s)} \ln \frac{W(x)}{W(x)-W(s)}\right)^{\beta}}{(W(x)-W(s))^{1-\alpha}} W^{\beta}(s) u(s) f(s) w(s) d s\right)^{q} d x \\
& \leq \sum_{k} \int_{x_{k}}^{x_{k+1}} v(x) \frac{\left(\frac{1}{W\left(x_{k-1}\right)} \ln \frac{W(x)}{\left(W(x)-W\left(x_{k-1}\right)\right.}\right)^{q \beta}}{\left(W(x)-W\left(x_{k-1}\right)\right)^{q(1-\alpha)}}\left(\int_{0}^{x_{k-1}} W^{\beta}(s) u(s) f(s) d s\right)^{q} d x .
\end{aligned}
$$

Since $W\left(x_{k-1}\right)=2^{k-1}=\frac{1}{2} W\left(x_{k}\right) \leq \frac{1}{2} W(x)$ and $W\left(x_{k-1}\right)=\frac{1}{4} W\left(x_{k+1}\right) \geq \frac{1}{4} W(x)$ for $x_{k} \leq x \leq$ $x_{k+1}$, then from (3.4) it follows

$$
J_{1} \leq 2^{-(2 q \beta+q(1-\alpha))} \ln ^{q \beta} 2\left(\int_{0}^{\infty} v(x) W^{q(\alpha-\beta-1)}(x)\left(\int_{0}^{x} W^{\beta}(s) u(s) f(s) w(s) d s\right)^{q} d x\right) .
$$

Hence, based on Theorem A,

$$
J_{1} \ll A_{\alpha, \beta}^{q}\|f\|_{p, w}^{q}
$$

Now, we estimate $J_{2}$. Using the nonincreasing function $u$ for estimating $J_{2}$ and applying Hölder's inequality, we find

$$
\begin{aligned}
J_{2}= & \sum_{k} \int_{x_{k}}^{x_{k+1}} v(x)\left(\int_{x_{k-1}}^{x} \frac{\left(\ln \frac{W(x)}{W(x)-W(s)}\right)^{\beta}}{(W(x)-W(s))^{1-\alpha}} u(s) f(s) w(s) d s\right)^{q} d x \\
= & \sum_{k} \int_{x_{k}}^{x_{k+1}} v(x) W^{-q \beta}(x)\left(\int_{x_{k-1}}^{x} \frac{\left(\frac{W(x)}{W(s)} \ln \frac{W(x)}{W(x)-W(s)}\right)^{\beta}}{(W(x)-W(s))^{1-\alpha}} W^{\beta}(s) u(s) f(s) w(s) d s\right)^{q} d x \\
\leq & \sum_{k} u^{q}\left(x_{k-1}\right) W^{q \beta}\left(x_{k+1}\right) \\
& \times \int_{x_{k}}^{x_{k+1}} v(x) W^{-q \beta}(x)\left(\int_{x_{k-1}}^{x} \frac{\left(\frac{W(x)}{W(s)} \ln \frac{W(x)}{W(x)-W(s)}\right)^{\beta}}{(W(x)-W(s))^{1-\alpha}} f(s) w(s) d s\right)^{q} d x \\
\leq & \sum_{k} u^{q}\left(x_{k-1}\right) W^{q \beta}\left(x_{k+1}\right) \int_{x_{k}}^{x_{k+1}} v(x) W^{-q \beta}(x)\left(\int_{x_{k-1}}^{x} \frac{(W(x)}{W(s)} \ln \frac{W(x)}{W(x)-W(s)}\right)^{p^{\prime} \beta} \\
& \left.\times\left(\int_{x_{k-1}}^{x_{k+1}} f^{p}(s) w(s)\right)^{p^{\prime}(1-\alpha)} d s\right)^{\frac{q}{p^{\prime}}} d x .
\end{aligned}
$$

We replace the variables $W(s)=W(x) t$ in the following expression:

$$
\begin{aligned}
& \int_{x_{k-1}}^{x} \frac{\left(\frac{W(x)}{W(s)} \ln \frac{W(x)}{W(x)-W(s)}\right)^{p^{\prime} \beta}}{(W(x)-W(s))^{p^{\prime}(1-\alpha)}} w(s) d s \\
& \quad=W^{p^{\prime}(\alpha-1)+1}(x) \int_{2^{k-1} W^{-1}(x)}^{1} \frac{\left(\frac{1}{t} \ln \frac{1}{1-t}\right)^{p^{\prime} \beta}}{(1-t) p^{\prime}(1-\alpha)} d t \\
& \quad \leq W\left(x_{k+1}\right) W^{p^{\prime}(\alpha-1)}(x) \int_{2^{k-1} W^{-1}\left(x_{k+1}\right)}^{1} \frac{\left(\frac{1}{t} \ln \frac{1}{1-t}\right)^{p^{\prime} \beta}}{(1-t)^{p^{\prime}(1-\alpha)}} d t
\end{aligned}
$$




$$
\begin{aligned}
& \leq 2^{2 p^{\prime} \beta} W\left(x_{k+1}\right) W^{p^{\prime}(\alpha-1)}(x) \int_{0}^{1} \frac{\left(\ln \frac{1}{1-t}\right)^{p^{\prime} \beta}}{(1-t)^{p^{\prime}(1-\alpha)}} d t \\
& =\gamma 2^{2 p^{\prime} \beta} W\left(x_{k+1}\right) W^{p^{\prime}(\alpha-1)}(x)
\end{aligned}
$$

for $x_{k} \leq x \leq x_{k+1}$, where $\gamma=\int_{0}^{1} \frac{\left(\ln \frac{1}{1-t} p^{p^{\prime} \beta}\right.}{(1-t)^{p^{\prime}(1-\alpha)}} d t=\int_{1}^{\infty} z^{p^{\prime} \beta} e^{-z p^{\prime}\left(\alpha-\frac{1}{p}\right)} d z<\infty$.

In the latter ratio, we used a replacement $\frac{1}{1-t}=e^{z}$. Substituting the obtained estimates (3.7) in (3.6), we get

$$
\begin{aligned}
J_{2} \ll & \sum_{k} u^{q}\left(x_{k-1}\right) W^{q \beta+\frac{q}{p^{\prime}}}\left(x_{k+1}\right) \\
& \times \int_{x_{k}}^{x_{k+1}} v(x) W^{q(\alpha-\beta-1)}(x)\left(\int_{x_{k-1}}^{x_{k+1}}|f(s)|^{p} w(s) d s\right)^{\frac{q}{p}} d x .
\end{aligned}
$$

Next, we need the following estimation:

$$
\begin{aligned}
u^{q}\left(x_{k-1}\right) W^{q \beta+\frac{q}{p^{\prime}}}\left(x_{k+1}\right) & =2^{2\left(q \beta+\frac{q}{p^{\prime}}\right)}\left(u^{p^{\prime}}\left(x_{k-1}\right) W^{p^{\prime} \beta+1}\left(x_{k-1}\right)\right)^{\frac{q}{p^{\prime}}} \\
& =2^{2 q\left(\beta+\frac{1}{p^{\prime}}\right)}\left(p^{\prime} \beta+1\right)^{\frac{q}{p^{\prime}}}\left(u^{p^{\prime}}\left(x_{k-1}\right) \int_{0}^{x_{k-1}} W^{p^{\prime} \beta}(s) w(s) d s\right)^{\frac{q}{p^{\prime}}} \\
& \ll\left(\int_{0}^{x_{k-1}} u^{p^{\prime}}(s) W^{p^{\prime} \beta}(s) w(s) d s\right)^{\frac{q}{p^{\prime}}} .
\end{aligned}
$$

Substituting the obtained estimate in (3.8) and using Jensen's inequality, by virtue of $p \leq q$, we have

$$
\begin{aligned}
J_{2} \ll & \sum_{k}\left(\int_{0}^{x_{k-1}} u^{p^{\prime}}(s) W^{p^{\prime} \beta}(s) w(s) d s\right)^{\frac{q}{p^{\prime}}}\left(\int_{x_{k-1}}^{\infty} v(x) W^{q(\alpha-\beta-1)}(x) d x\right) \\
& \times\left(\int_{x_{k-1}}^{x_{k+1}}|f(s)|^{p} w(s) d s\right)^{\frac{q}{p}} \\
\leq & A_{\alpha, \beta}^{q} \sum_{k}\left(\int_{x_{k-1}}^{x_{k+1}}|f(s)|^{p} w(s) d s\right)^{\frac{q}{p}} d x \ll A_{\alpha, \beta}^{q}\|f\|_{p, w}^{q} .
\end{aligned}
$$

Substituting the obtained estimates (3.5) and (3.9) in (3.3), we get

$$
\left\|T_{\alpha, \beta} f\right\|_{q, v} \ll A_{\alpha, \beta}\|f\|_{p, w},
$$

i.e., the boundedness of the operator $T_{\alpha, \beta}$ from $L_{p, w}$ to $L_{q, v}$ and the estimate $\left\|T_{\alpha, \beta}\right\| \ll A_{\alpha, \beta}$ holds for the norm $\left\|T_{\alpha, \beta}\right\|$ from $L_{p, w}$ to $L_{q, v}$, which together with (3.2) gives $\left\|T_{\alpha, \beta}\right\| \approx A_{\alpha, \beta}$. Theorem 3.1 is proved.

\section{The compactness of the operator $T_{\alpha, \beta}$}

Assume that

$$
A_{\alpha, \beta}(z)=\left(\int_{z}^{\infty} v(x) W^{q(\alpha-\beta-1)}(x) d x\right)^{\frac{1}{q}}\left(\int_{0}^{z} W^{\beta p^{\prime}}(s) u^{p^{\prime}}(s) w(s) d s\right)^{\frac{1}{p^{\prime}}} .
$$


Theorem 4.1 Let $0<\alpha<1, \frac{1}{\alpha}<p \leq q<\infty$, and $\beta \geq 0$. Let the function $u$ be nonincreasing on the interval I. Then the operator $T_{\alpha, \beta}$ is compact from $L_{p, w}$ to $L_{q, v}$ if and only if $A_{\alpha, \beta}<\infty$ and

$$
\lim _{z \rightarrow 0^{+}} A_{\alpha, \beta}(z)=\lim _{z \rightarrow \infty} A_{\alpha, \beta}(z)=0 .
$$

Proof of Theorem 4.1 Necessity. Let the operator $T_{\alpha, \beta}$ be compact from $L_{p, w}$ to $L_{q, v}$. Then it is bounded from $L_{p, w}$ to $L_{q, v}$ and $A_{\alpha, \beta}<\infty$ according to Theorem 3.1. First, let us show the fulfilment of $\lim _{z \rightarrow 0^{+}} A_{\alpha, \beta}(z)=0$. Consider the family of functions $\left\{f_{t}\right\}_{t \in I}$ :

$$
f_{t}(x)=\chi_{(0, t)}(x) u^{p^{\prime}-1}(x) W^{\left(p^{\prime}-1\right) \beta}(x)\left(\int_{0}^{t} u^{p^{\prime}}(s) W^{\beta p^{\prime}}(s) w(s) d s\right)^{-\frac{1}{p}}, \quad x, t \in I .
$$

Let us note that

$$
\begin{aligned}
\left\|f_{t}\right\|_{p, w} & =\left(\int_{0}^{t}\left|f_{t}(x)\right|^{p} w(x) d x\right)^{\frac{1}{p}} \\
& =\left(\int_{0}^{t} u^{p^{\prime}}(x) W^{\beta p^{\prime}}(x) w(x) d x\right)^{\frac{1}{p}}\left(\int_{0}^{t} u^{p^{\prime}}(s) W^{\beta p^{\prime}}(s) w(s) d s\right)^{-\frac{1}{p}}=1,
\end{aligned}
$$

i.e., $f_{t} \in L_{p, w}$ for all $t \in I$. Let us show that $f_{t}$ converges weakly to zero if $t \rightarrow 0^{+}$. For arbitrary $g \in\left(L_{p, w}\right)^{*}=L_{p^{\prime}, w^{1-p^{\prime}}}$, we have

$$
\begin{aligned}
\int_{0}^{\infty} f_{t}(x) g(x) d x & \leq\left(\int_{0}^{t}\left|f_{t}(x)\right|^{p} w(x) d x\right)^{\frac{1}{p}}\left(\int_{0}^{t}|g(s)|^{p^{\prime}} w^{1-p^{\prime}}(s) d s\right)^{\frac{1}{p^{\prime}}} \\
& =\left(\int_{0}^{t}|g(s)|^{p^{\prime}} w^{1-p^{\prime}}(s) d s\right)^{\frac{1}{p}} .
\end{aligned}
$$

Whence it follows that $f_{t}$ weakly converges to zero if $t \rightarrow 0^{+}$. Since the operator $T_{\alpha, \beta}$ is compact from $L_{p, w}$ to $L_{q, v}$, then

$$
\lim _{t \rightarrow 0^{+}}\left\|T_{\alpha, \beta} f_{t}\right\|_{q, v}=0
$$

We have

$$
\begin{aligned}
\left\|T_{\alpha, \beta} f_{t}\right\|_{q, v}^{q} & \geq \int_{t}^{\infty} v(x)\left(\int_{0}^{t} \frac{\left(\ln \frac{W(x)}{W(x)-W(s)}\right)^{\beta}}{(W(x)-W(s))^{1-\alpha}} u(s) f_{t}(s) w(s) d s\right)^{q} d x \\
& \geq \int_{t}^{\infty} v(x) W^{q(\alpha-\beta-1)}(x)\left(\int_{0}^{t} W^{\beta}(s) u(s) f_{t}(s) w(s) d s\right)^{q} d x \\
& =\int_{t}^{\infty} v(x) W^{q(\alpha-\beta-1)}(x) d x\left(\int_{0}^{t} W^{p^{\prime} \beta}(s) u^{p^{\prime}}(s) w(s) d s\right)^{\frac{q}{p^{\prime}}}=\left(A_{\alpha, \beta}(t)\right)^{\frac{1}{q}} .
\end{aligned}
$$

Whence and from (4.2) it follows that $\lim _{t \rightarrow 0^{+}} A_{\alpha, \beta}(t)=0$. We now prove that $\lim _{t \rightarrow \infty} A_{\alpha, \beta}(t)=0$. The compactness of the adjoint operator

$$
T_{\alpha, \beta}^{*} g(s)=u(s) w(s) \int_{s}^{\infty} \frac{\left(\ln \frac{W(x)}{W(x)-W(s)}\right)^{\beta}}{(W(x)-W(s))^{1-\alpha}} g(x) d x
$$


from $L_{q^{\prime}, v^{1-q^{\prime}}}$ to $L_{p^{\prime}, w^{1-p^{\prime}}}$ follows from the compactness of the operator $T_{\alpha, \beta}$ from $L_{p, w}$ to $L_{q, v}$.

Introduce the family of functions $\left\{g_{t}\right\}_{t \in I}$ :

$$
g_{t}(x)=\chi_{(t, \infty)}(x) W^{(q-1)(\alpha-\beta-1)}(x) v(x)\left(\int_{t}^{\infty} W^{q(\alpha-\beta-1)}(s) v(s) d s\right)^{-\frac{1}{q^{\prime}}} .
$$

It is easy to see that $g_{t} \in L_{q^{\prime}, v^{1-q^{\prime}}}$ for all $t \in I$. Indeed,

$$
\begin{aligned}
\left\|g_{t}\right\|_{q^{\prime}, v^{1-q^{\prime}}} & =\left(\int_{t}^{\infty}\left|W^{(q-1)(\alpha-\beta-1)}(x) v(x)\right|^{q^{\prime}} v^{1-q^{\prime}}(x) d x\right)^{\frac{1}{q^{\prime}}}\left(\int_{t}^{\infty} W^{q(\alpha-\beta-1)}(s) v(s) d s\right)^{-\frac{1}{q^{\prime}}} \\
& =1 .
\end{aligned}
$$

Let $f \in\left(L_{q^{\prime}, v^{1-q^{\prime}}}\right)^{*}=L_{q, v}$ be an arbitrary function. Then

$$
\begin{aligned}
\int_{0}^{\infty} g_{t}(x) f(x) d x & \leq\left(\int_{t}^{\infty}\left|g_{t}(x)\right|^{q^{\prime}} v^{1-q^{\prime}}(x) d x\right)^{\frac{1}{q^{\prime}}}\left(\int_{t}^{\infty}|f(s)|^{q} v(s) d s\right)^{\frac{1}{q}} \\
& =\left(\int_{t}^{\infty}|f(x)|^{q} v(x) d x\right)^{\frac{1}{q}} .
\end{aligned}
$$

This implies that $\lim _{t \rightarrow \infty} \int_{0}^{\infty} g_{t}(x) f(x) d x=0$ for all $f \in L_{q, v}$. Consequently, the family of functions $\left\{g_{t}\right\}_{t \in I} \subset L_{q^{\prime}, v^{1-q^{\prime}}}$ weakly converges to zero at $t \rightarrow \infty$.

Then, from the compactness $T_{\alpha, \beta}^{*}: L_{q^{\prime}, v^{1-q^{\prime}}} \rightarrow L_{p^{\prime}, w^{1-p^{\prime}}}$, we have

$$
\lim _{t \rightarrow \infty}\left\|T_{\alpha, \beta}^{*} g_{t}\right\|_{p^{\prime}, w^{1-p^{\prime}}}=0
$$

Since

$$
\begin{aligned}
\left\|T_{\alpha, \beta}^{*} g_{t}\right\|_{p^{\prime}, w^{1-p^{\prime}}} \geq & \left(\int_{0}^{t} u^{p^{\prime}}(s) w(s)\left(\int_{t}^{\infty} \frac{\left(\ln \frac{W(x)}{W(x)-W(s)}\right)^{\beta}}{(W(x)-W(s))^{1-\alpha}} g_{t}(x) d x\right)^{p^{\prime}} d s\right)^{\frac{1}{p^{\prime}}} \\
\geq & \left(\int_{0}^{t} u^{p^{\prime}}(s) W^{p^{\prime} \beta}(s) w(s)\left(\int_{t}^{\infty} W^{(\alpha-\beta-1)}(x) g_{t}(x) d x\right)^{p^{\prime}} d s\right)^{\frac{1}{p^{\prime}}} \\
= & \left(\int_{0}^{t} u^{p^{\prime}}(s) W^{p^{\prime} \beta}(s) w(s) d s\right)^{\frac{1}{p^{\prime}}}\left(\int_{t}^{\infty} W^{q(\alpha-\beta-1)}(x) v(x) d x\right) \\
& \times\left(\int_{t}^{\infty} W^{q(\alpha-\beta-1)}(x) v(x) d x\right)^{-\frac{1}{q^{\prime}}}=A_{\alpha, \beta}(t),
\end{aligned}
$$

then (4.3) implies that $\lim _{t \rightarrow \infty} A_{\alpha, \beta}(t)=0$. The necessity has been proven.

Sufficiency. Let $A_{\alpha, \beta}<\infty$ and (4.1) be fulfilled. We define $P_{c} f=\chi_{(0, c]} f, P_{c d} f=\chi_{(c, d l} f$ and $Q_{d} f=\chi_{(d, \infty)} f$ for $0<c<d<\infty$. Then $f=P_{c} f+P_{c d} f+Q_{d} f$ and, by virtue of $P_{c} T_{\alpha, \beta} P_{c d} \equiv 0$, $P_{c} T_{\alpha, \beta} Q_{d} \equiv 0$ and $P_{c d} T_{\alpha, \beta} Q_{d} \equiv 0$, we obtain

$$
T_{\alpha, \beta} f=P_{c d} T_{\alpha, \beta} P_{c d} f+P_{c} T_{\alpha, \beta} P_{c} f+P_{c d} T_{\alpha, \beta} P_{c} f+Q_{d} T_{\alpha, \beta} f .
$$

Let us show that the operator $P_{c d} T_{\alpha, \beta} P_{c d}$ is compact from $L_{p, w}$ to $L_{q, v}$. Since $P_{c d} T_{\alpha, \beta} P_{c d} \times$ $f(x)=0$ for $x \in I \backslash(c, d)$, then it suffices to show that the operator is compact from $L_{p, w}(c, d)$ 
to $L_{q, v}(c, d)$. This is equivalent to the compactness of the operator

$$
T f(x)=\int_{c}^{d} K(x, s) f(s) d s
$$

from $L_{p}(c, d)$ to $L_{q}(c, d)$ with the kernel

$$
K(x, s)=u(s) v^{\frac{1}{q}}(x) \chi_{(c, d)}(x-s) w^{\frac{1}{p^{\prime}}}(s) \frac{\left(\ln \frac{W(x)}{W(x)-W(s)}\right)^{\beta}}{(W(x)-W(s))^{1-\alpha}} .
$$

Let $\left\{x_{k}\right\}_{k \in Z}$ be a sequence constructed by the function $W$ from Theorem 3.1. Then there exist numbers $i$ and $n$ such that $x_{i} \leq c<x_{i+1}, x_{n}<d \leq x_{n+1}$. We will assume that the numbers $c, d$ are chosen so that $x_{i+1}<x_{n}$. Proceeding as in Theorem 3.1, we have

$$
\begin{aligned}
& \int_{c}^{d}\left(\int_{c}^{d}|K(x, s)|^{p^{\prime}} d s\right)^{\frac{q}{p^{\prime}}} d x \\
& \quad \leq \sum_{k=i}^{n} \int_{x_{k}}^{x_{k+1}} v(x)\left[\left(\int_{0}^{x_{k-1}}+\int_{x_{k-1}}^{x}\right) \frac{\left(\ln \frac{W(x)}{W(x)-W(s)}\right)^{p^{\prime} \beta}}{\left(W(x)-W(s) p^{p^{\prime}(1-\alpha)}\right.} u^{p^{\prime}}(s) w(s) d s\right]^{\frac{q}{p^{\prime}}} d x \\
& \quad \ll F_{1}+F_{2} .
\end{aligned}
$$

Estimate $F_{1}$ and $F_{2}$. Analogously to the estimate of $J_{1}$,

$$
\begin{aligned}
F_{1} & =\sum_{k=i}^{n} \int_{x_{k}}^{x_{k+1}} v(x)\left(\int_{0}^{x_{k-1}} \frac{\left(\ln \frac{W(x)}{W(x)-W(s)}\right)^{p^{\prime} \beta}}{(W(x)-W(s))^{p^{\prime}(1-\alpha)}} u^{p^{\prime}}(s) w(s) d s\right)^{\frac{q}{p^{\prime}}} d x \\
& \leq \sum_{k=i}^{n} \int_{x_{k}}^{x_{k+1}} v(x) \frac{\left(\frac{1}{W\left(x_{k-1}\right)} \ln \frac{W(x)}{\left(W(x)-W\left(x_{k-1}\right)\right.}\right)^{q \beta}}{\left(W\left(x_{k-1}\right)\right)^{q(1-\alpha)}} d x\left(\int_{0}^{x_{k-1}} W^{p^{\prime} \beta}(s) u^{p^{\prime}}(s) w(s) d s\right)^{\frac{q}{p^{\prime}}} \\
& \ll \sum_{k=i}^{n} A_{\alpha, \beta}^{q}\left(x_{k-1}\right) \leq(n-i+1) A_{\alpha, \beta}^{q} .
\end{aligned}
$$

Analogously to the estimate of $J_{2}$,

$$
\begin{aligned}
F_{2} & =\sum_{k=i}^{n} \int_{x_{k}}^{x_{k+1}} v(x)\left(\int_{x_{k-1}}^{x} \frac{\left(\ln \frac{W(x)}{W(x)-W(s)}\right)^{p^{\prime} \beta}}{(W(x)-W(s))^{p^{\prime}(1-\alpha)}} u^{p^{\prime}}(s) w(s) d s\right)^{\frac{q}{p^{\prime}}} d x \\
& \ll \sum_{k=i}^{n} u^{q}\left(x_{k-1}\right) W^{q\left(\beta+\frac{1}{p^{\prime}}\right)}\left(x_{k-1}\right) \int_{x_{k}}^{x_{k+1}} v(x) W^{q(\alpha-\beta-1)}(x) d x\left(\int_{0}^{1} \frac{\left(\ln \frac{1}{1-t}\right)^{p^{\prime} \beta}}{(1-t)^{p^{\prime}(1-\alpha)}} d t\right)^{\frac{q}{p^{\prime}}} \\
& \ll(n-i+1) A_{\alpha, \beta} .
\end{aligned}
$$

Substituting (4.6) and (4.7) into (4.5), we obtain

$$
\int_{c}^{d}\left(\int_{c}^{d}|K(x, s)|^{p^{\prime}} d s\right)^{\frac{q}{p^{\prime}}} d x<\infty
$$

Therefore, based on the Kantorovich criterion ([11], XI, paragraph 3) the operator $T$ is compact from $L_{p}(c, d)$ to $L_{q}(c, d)$, which is equivalent to the compactness of the operator 
$P_{c d} T_{\alpha, \beta} P_{c d}$ from $L_{p, w}$ to $L_{q, v}$. From (4.4) we have

$$
\left\|T_{\alpha, \beta}-P_{c d} T_{\alpha, \beta} P_{c d}\right\| \leq\left\|P_{c} T_{\alpha, \beta} P_{c}\right\|+\left\|P_{c d} T_{\alpha, \beta} P_{c}\right\|+\left\|Q_{d} T_{\alpha, \beta}\right\|
$$

Further, we assume that the right-hand side of (4.8) tends to zero as $c \rightarrow 0$ and $d \rightarrow$ $\infty$. Then the operator $T_{\alpha, \beta}$ is compact from $L_{p, w}$ to $L_{q, v}$ as the uniform limit of compact operators.

Based on Theorem 3.1, we obtain

$$
\begin{aligned}
\left\|P_{c} T_{\alpha, \beta} P_{c} f\right\|_{q, v} & =\left(\int_{0}^{\infty} P_{c} v(x)\left|T_{\alpha, \beta} P_{c} f(x)\right|^{q} d x\right)^{\frac{1}{q}} \\
& \ll \sup _{a<z<c} A_{\alpha, \beta}(z)\|f\|_{p, w} .
\end{aligned}
$$

Therefore, $\left\|P_{c} T_{\alpha, \beta} P_{c}\right\| \ll \sup _{a<z<c} A_{\alpha, \beta}(z)$.

Whence and from $\lim _{z \rightarrow 0^{+}} A_{\alpha, \beta}(z)=0$ it follows that

$$
\begin{aligned}
\lim _{c \rightarrow 0^{+}}\left\|P_{c} T_{\alpha, \beta} P_{c}\right\| & =0 ; \\
\left\|P_{c d} T_{\alpha, \beta} P_{c} f\right\|_{q, v} & =\left(\int_{0}^{\infty} P_{c d} v(x)\left|T_{\alpha, \beta} P_{c} f(x)\right|^{q} d x\right)^{\frac{1}{q}} \\
& \ll A_{\alpha, \beta}(c, d)\|f\|_{p, w},
\end{aligned}
$$

where

$$
\begin{aligned}
A_{\alpha, \beta}(c, d) & =\sup _{z>0}\left(\int_{z}^{\infty} P_{c d} v(x) W^{q(\alpha-\beta-1)}(x) d x\right)^{\frac{1}{q}}\left(\int_{0}^{z} W^{p^{\prime} \beta}(s) P_{c} u^{p^{\prime}}(s) w(s) d s\right)^{\frac{1}{p^{\prime}}} \\
& =\sup _{0<z<d} A_{\alpha, \beta}(c, d)=A_{\alpha, \beta}(c) .
\end{aligned}
$$

Therefore $\left\|P_{c d} T_{\alpha, \beta} P_{c}\right\| \ll A_{\alpha, \beta}(c)$ and whence

$$
\lim _{c \rightarrow 0^{+}}\left\|P_{c d} T_{\alpha, \beta} P_{c}\right\|=0
$$

holds.

Similarly, we have

$$
\begin{aligned}
\left\|Q_{d} T_{\alpha, \beta} f\right\|_{q, v} & =\left(\int_{0}^{\infty} Q_{d} v(x)\left|T_{\alpha, \beta} f(x)\right|^{q} d x\right)^{\frac{1}{q}} \\
& \ll \sup _{z>d} A_{\alpha, \beta}(z)\|f\|_{p, w} .
\end{aligned}
$$

and $\left\|Q_{d} T_{\alpha, \beta}\right\| \ll \sup _{z>d} A_{\alpha, \beta}(z)$.

From this and from $\lim _{z \rightarrow \infty} A_{\alpha, \beta}(z)=0$, we obtain

$$
\lim _{d \rightarrow \infty}\left\|Q_{d} T_{\alpha, \beta}\right\|=0
$$

From (4.8), (4.9), (4.10), and (4.11) it follows that the operator $T_{\alpha, \beta}$ is compact from $L_{p, w}$ to $L_{q, v}$. Theorem 4.1 is completely proved. 


\section{Consequences}

When $W(x)=x$ the operator $T_{\alpha, \beta}$ has the form

$$
J_{\alpha, \beta} f(x)=\int_{0}^{x} \frac{\left(\ln \frac{x}{x-s}\right)^{\beta}}{(x-s)^{1-\alpha}} u(s) f(s) d s, \quad x>0 .
$$

Note that the operator

$$
J f(x)=\int_{0}^{x} \ln \frac{x}{x-s} f(s) \frac{d s}{s}
$$

is called [12] the infinitesimal order fractional integration operator.

From Theorems 3.1 and 4.1, as a consequence, we have the following.

Corollary 5.1 Let $0<\alpha<1, \frac{1}{\alpha}<p \leq q<\infty$, and $\beta \geq 0$. Let the function u be nonincreasing on $I$. Then the operator $J_{\alpha, \beta}$ is bounded from $L_{p}$ to $L_{q, v}$ if and only if $A_{\alpha, \beta}=\sup _{z>0} A_{\alpha, \beta}(z)<$ $\infty$, where

$$
A_{\alpha, \beta}(z)=\left(\int_{z}^{\infty} v(x) x^{q(\alpha-\beta-1)}(x) d x\right)^{\frac{1}{q}}\left(\int_{0}^{z} s^{\beta p^{\prime}} u^{p^{\prime}}(s) d s\right)^{\frac{1}{p^{\prime}}},
$$

wherein $\left\|J_{\alpha, \beta}\right\| \approx A_{\alpha, \beta}$, where $\left\|J_{\alpha, \beta}\right\|$ is the norm of the operator $J_{\alpha, \beta}$ from $L_{p}$ to $L_{q, v}$.

Corollary 5.2 Let $0<\alpha<1, \frac{1}{\alpha}<p \leq q<\infty$, and $\beta \geq 0$. Let the function $u$ be nonincreasing on $I$. Then the operator $J_{\alpha, \beta}$ is compact from $L_{p}$ to $L_{q, v}$ if and only if $A_{\alpha, \beta}<\infty$ and

$$
\lim _{z \rightarrow 0^{+}} A_{\alpha, \beta}(z)=\lim _{z \rightarrow \infty} A_{\alpha, \beta}(z)=0 .
$$

Note that the boundedness and compactness of the operator

$$
J_{\alpha}^{\prime} f(x)=\int_{0}^{x}(x-s)^{\alpha-1}\left(\ln \frac{\gamma}{x-s}\right)^{\beta} f(s) d s
$$

from $L_{p}(0, a)$ to $L_{q, v}(0, a)$ or from $L_{p, v}(0, a)$ to $L_{q}(0, a)$ were established in [13], where $0<a \leq$ $\gamma<\infty, \alpha>\frac{1}{p}$.

\section{Acknowledgements}

We thank both referees for very good remarks, which have helped us to improve the final version of this paper.

\section{Funding}

This paper was supported by the grant of the Ministry of Education and Science of the Republic of Kazakhstan [grant number (IRN): AP08856339].

\section{Availability of data and materials} Not applicable.

\section{Declarations}

\section{Competing interests}

The authors declare that they have no competing interests.

\section{Authors' contributions}

AA: conceptualization, investigation, writing-original draft, writing - review and editing, funding acquisition. RO: problem statement, conceptualization, methodology, investigation, writing —original draft, supervision. BS: investigation, writing - review and editing. All the authors read and approved the final manuscript. 


\section{Publisher's Note}

Springer Nature remains neutral with regard to jurisdictional claims in published maps and institutional affiliations.

Received: 29 September 2021 Accepted: 27 January 2022 Published online: 10 February 2022

\section{References}

1. Andersen, K.F., Sawyer, E.T.: Weighted norm inequalities for the Riemann-Liouville and Weyl fractional integral operators. Trans. Am. Math. Soc. 308(2), 547-558 (1988)

2. Meskhi, A.: Solution of some weight problems for the Riemann-Liouville and Weil operators. Georgian Math. J. 5(6), 565-574 (1998)

3. Prokhorov, D.V.: On the boundedness and compactness of a class of integral operators. J. Lond. Math. Soc. 61(2), 617-628 (2000)

4. Prokhorov, D.V., Stepanov, V.D.: Weighted estimates for the Riemann-Liouville operators and applications. Tr. Mat. Inst. Steklova 243, 289-312 (2003) (in Russian)

5. Abylayeva, A.M., Oinarov, R., Persson, L.E.: Boundedness and compactness of a class of Hardy type operators. J. Inequal. Appl. 2016, 324 (2016)

6. Oinarov, R:: Two-sided estimates of the norms for certain classes of integral operators. Proc. Math. Inst. RAS Steklov V.A. 204, 240-250 (1993) (in Russian)

7. Abylayeva, A.M.: Boundedness, compactness for a class of fractional integral operators of Weyl type. Eurasian Math. J. 7(1), 9-27 (2016)

8. Abylayeva, A.M.: Boundedness and compactness of the Hardy type operator with variable upper limit in weighted Lebesgue spaces. Math. Inequal. Appl. 23(3), 805-819 (2020)

9. Abylayeva, A.M., Persson, L.E.: Hardy type inequalities and compactness of a class of integral operators with logarithmic singularities. Math. Inequal. Appl. 21(1), 201-215 (2018)

10. Kufner, A., Maligranda, L., Persson, L.E.: The Hardy Inequality. About Its History and Some Related Results. Vydavatelsky Servis Publishing House, Pilsen (2007)

11. Kantarovich, L.V., Akilov, G.R.: Functional Analysis. Nauka, Moscow (1977) (in Russian)

12. Nakhushev, A.M.: Equations of Mathematical Biology. M. Vysshaya Shkola, Moscow (1995) (in Russian)

13. Kokilashvili, V., Meskhi, A.: Criteria for the boundedness and compactness of operators with power-logarithmic kernels. Anal. Math. 27, 173-185 (2001)

\section{Submit your manuscript to a SpringerOpen ${ }^{\circ}$ journal and benefit from:}

- Convenient online submission

- Rigorous peer review

- Open access: articles freely available online

- High visibility within the field

- Retaining the copyright to your article

Submit your next manuscript at $\boldsymbol{\nabla}$ springeropen.com 